\title{
THE RESULTS OF COLLAPSE AND CONSERVATIVE THERAPY IN PULMONARY TUBERCULOSIS *
}

\author{
BY
}

\author{
A. F. FOSTER-CARTER, M. MYERS, D. L. H. GODDARD, F. H. YOUNG, \\ AND B. BENJAMIN \\ From the Brompton Hospital Sanatorium, Frimley
}

(RECEIVED FOR PUblication JANUARY 31, 1953)

The survey comprised 1,153 patients discharged from the sanatorium during the five-year period 1937 to 1942. All patients were followed for at least eight years and only $1 \%$ were lost from observation.

The radiological extent of disease on admission to hospital was classified as follows: Class I, one zone ; Class II, two or three zones ; Class III, four or more zones. The expectation of survival was found to be inversely proportional to the extent of disease, regardless of other factors. Care was therefore taken to see that the distribution of extent of disease was similar in groups of patients used for comparisons.

Of the 955 patients with cavitated disease, 311 . had no initial collapse therapy, of whom $50 \%$ survived ; 256 had no collapse therapy at any time, and $46 \%$ of them survived eight years ; those with advanced disease (Class III) had a survival rate of $33 \%$, while $74 \%$ of those in Classes I and II survived. A consistently negative sputum was a good prognostic factor, regardless of the extent of disease, and sputum conversion also improved the prognosis. The extent of disease was a more important factor than the size of cavity. One hundred and twenty patients had large cavities $(2.5 \mathrm{~cm}$. or more in diameter) and $28 \%$ of them survived eight years without active treatment ; 66 had sanatorium treatment, including graduated exercise ; $45 \%$ of these were alive and $32 \%$ were working at the end of eight years. Sixteen per cent. of the original 311 relapsed and had to have collapse therapy; most relapses occurred within the first two years, but they continued in appreciable numbers up to six years.

Six hundred and forty-four patients had some form of collapse therapy as initial treatment (71\% of these had a unilateral pneumothorax and $12 \%$ had a thoracoplasty); $67 \%$ of all these

\footnotetext{
*This paper is an abstract of a communication made by Dr. A. F Foster-Carter to the Thoracic Society in February, 1953. It is published in full in the Brompton Hospital Reports, 21, 1.
}

patients survived for eight years. In patients with disease of the same extent there was no difference in prognosis if the disease was unilateral or bilateral. The eight-year survival rate of Class III patients with large cavities was $47 \%$, more than double that of similar patients who had no collapse therapy.

Four hundred and fifty-seven patients had unilateral pneumothorax treatment; they were divided into three groups according to the type and efficiency of the collapse:

SATisfactory.- Those who had a free lung (92. cases).

AdHerent-Satisfactory.-Those who had adhesions over the diseased area but whose cavities closed within a year (242 cases).

AdHerent-UnSaTisfactory.-Those who had adhesions over the diseased area with a persistent cavity and had either developed an empyema or still had an unclosed cavity at the end of a year (113 cases).

The eight-year survival rates for these three groups were: Satisfactory, $79 \%$, adherent-satisfactory, $82 \%$, adherent-unsatisfactory, $36 \%$.

A pneumothorax, with or without adhesions, which closed the cavity resulted in a longer survival, whatever the extent of disease, compared with those patients who had no collapse therapy. "Satisfactory" and "adherent-satisfactory" pneumothoraces resulted in the sputum becoming negative in over $80 \%$ of sputum-positive patients. The percentage of patients who required further collapse therapy at a later date was 37 in the "satisfactory" group and 30 in the "adherentsatisfactory" group. The presence of adhesions was not associated with an increased liability to develop pleural fluid, provided that the cavity had closed ; $36 \%$ of the patients in the "satisfactory" and "adherent-satisfactory" groups developed fluid, compared with $63 \%$ of those in the 
"adherent-unsatisfactory" group. Clear effusions were found in $26 \%$ of all patients and had little effect on the prognosis. Empyema occurred in $17 \%$ of all cases, but in patients whose cavities closed ("satisfactory" and "adherent-satisfactory") the incidence was $10 \%$ with a $35 \%$ mortality, while in those with persistent cavities (" adherent-unsatisfactory") the incidence was $36 \%$ and the mortality $73 \%$.

The majority of patients in the "adherentsatisfactory" group had numerous adhesions over the diseased area, but the degree of adherence did not affect the prognosis. Forty-five patients in this group had areas of lobar or segmental atelectasis, but this did not affect the outcome or the incidence of complications.

Provided that cavities closed, there was no statistical difference during the five years following the termination of pneumothorax treatment between patients who had adhesions and those who had free lungs. The highest five-year survival rate after re-expansion, $94 \%$, was found in a group of 89 patients who had undivided adhesions and whose pneumothoraces were terminated deliberately.

The general conclusion to be drawn from these 457 pneumothorax cases is that the closure of cavities, not absence of adhesions, is the key to successful pneumothorax treatment.

Seventy-seven patients were treated by thora- coplasty and $65 \%$ of them survived eight years. This figure does not include the operative mortality. Sputum-conversion was achieved in $49 \%$, and the 36 patients whose sputum became negative had an eight-year survival rate of $91 \%$. The results of thoracoplasty were statistically inferior to those of an "adherent-satisfactory" pneumothorax.

One hundred and ninety-eight patients who had pulmonary tuberculosis without obvious cavitation were followed for eight years. One hundred and thirty of these had no collapse therapy and their survival rate was $89 \%$. Only 12 had extensive disease. During the observation period $28 \%$ showed evidence of progressive disease and $18 \%$ required collapse therapy. Forty-one patients could be classed as "minimal" and their eightyear survival rate was $97 \%$; only four required collapse therapy.

Sixty-two patients with non-cavitated disease had unilateral pneumothorax treatment and $87 \%$ of them survived eight years. Only 10 had extensive disease, but 47 had persistent adhesions over the diseased area. The sputum conversion rate was $88 \%$, and $35 \%$ needed further collapse therapy at a later date. Thirty per cent. developed pleural fluid (clear $24 \%$, purulent $6 \%$ ). At the end of the observation period the treatment had been terminated about five years earlier in $85 \%$ of the survivors. 